University of Wollongong

Research Online

Faculty of Engineering - Papers (Archive)

Faculty of Engineering and Information

Sciences

July 2006

\title{
Nucleation, pinning, and coercivity in magnetic nanosystems: An analytical micromagnetic approach
}

\author{
G. P. Zhao \\ Sichuan Normal University, China \\ Xiaolin Wang \\ University of Wollongong, xiaolin@uow.edu.au
}

Follow this and additional works at: https://ro.uow.edu.au/engpapers

Part of the Engineering Commons

https://ro.uow.edu.au/engpapers/216

\section{Recommended Citation}

Zhao, G. P. and Wang, Xiaolin: Nucleation, pinning, and coercivity in magnetic nanosystems: An analytical micromagnetic approach 2006.

https://ro.uow.edu.au/engpapers/216

Research Online is the open access institutional repository for the University of Wollongong. For further information contact the UOW Library: research-pubs@uow.edu.au 


\title{
Nucleation, pinning, and coercivity in magnetic nanosystems: An analytical micromagnetic approach
}

\author{
G. P. Zhao ${ }^{1,2, *}$ and X. L. Wang ${ }^{2, \dagger}$ \\ ${ }^{1}$ Institute of Solid State Physics, Sichuan Normal University, Chengdu 610066, People's Republic of China \\ ${ }^{2}$ Institute for Superconducting and Electronic Materials, University of Wollongong, Northfields Avenue, \\ Wollongong, NSW 2522, Australia
}

(Received 29 May 2006; published 27 July 2006)

\begin{abstract}
Nucleation and pinning fields have been derived for an exchange-coupled hard/soft/hard magnetic nanosystem within an analytical micromagnetic approach. For the first time, the analytical formulas can be directly compared with the numerical and experimental coercivity to differentiate the coercivity mechanisms quantitatively. In addition, our analytical results demonstrate that the nucleation field decreases with the soft layer thickness $L^{s}$ parabolically, rather than reaching a plateau at small $L^{s}$ as claimed by other reports. The coercivity paradox and the attainable maximum energy product are reexamined based on our results.
\end{abstract}

DOI: 10.1103/PhysRevB.74.012409

PACS number(s): 75.60.Jk, 75.50.Ww, 75.70.Cn, 75.75.+a

The coercivity and its mechanism in permanent magnets has always been an intensive topic in magnetism. Since Brown's coercivity paradox, ${ }^{1}$ i.e., the measured coercivity is much lower than the theoretical value, was proposed in the 1940s, a lot of work has been devoted to the exploration of this problem. ${ }^{1-4}$ Nevertheless, the underlying details are still unclear. The phenomenological approach fits the experimental data quite well, but it cannot elucidate the underlying physics. The micromagnetic approach, in principle, can reveal the mechanism. To do that, the process from nucleation to coercivity has to be examined as a whole, with the associated nucleation and pinning fields derived explicitly. However, due to mathematical difficulties, in most previous studies, only the nucleation or pinning field has been obtained. As a result, so far the studies tackling the coercivity mechanism are mainly phenomenological.

Recently, an exchange-coupled composite material, proposed by Kneller in $1991,{ }^{5}$ with a hard phase to provide high coercivity and a soft phase to provide high saturation and remanence, aroused immediate interests from experts in this field. Skomski and Coey proposed in 1994 that the theoretical energy product, $(\mathrm{BH})_{\max }$, of oriented composite magnets could be as large as $1 \mathrm{MJ} / \mathrm{m}^{3}$, which almost doubles that of the best available single-phase permanent magnets. ${ }^{6}$ Many experiments have been carried out to realize this giant energy product over the past decade. The results, however, are quite discouraging since the best one so far is only about $0.2 \mathrm{MJ} / \mathrm{m}^{3}{ }^{7-10}$ It looks as if a new "energy product paradox" has emerged even before we could fully understand the coercivity paradox in this field.

In this paper, we seek to elucidate the coercivity mechanism within the framework of a self-contained micromagnetic model. The differential equations have been solved rigorously to provide reliable analytical formulas for nucleation and pinning fields for an exchange coupled magnetic triple system (see inset of Fig. 1). We chose such a structure because it appears to offer the best opportunity to realize the proposed giant energy product. On the other hand, the soft film can be regarded as a planar soft defect, which is normally present in single-phase hard magnetic materials. Thus the results can be applied in both permanent and composite magnets.
According to Brown, ${ }^{1}$ the total magnetic energy per unit area can be expressed as

$$
\begin{aligned}
\gamma= & \int_{0}^{L^{h}}\left[A^{h}\left[\frac{d \theta}{d z}\right]^{2}+K^{h} \sin ^{2} \theta-M_{S}^{h} H \cos \theta\right] d z \\
& +\int_{-L^{S} / 2}^{0}\left[A^{s}\left[\frac{d \theta}{d z}\right]^{2}+K^{s} \sin ^{2} \theta-M_{S}^{s} H \cos \theta\right] d z,
\end{aligned}
$$

where $A$ and $K$ are the exchange and anisotropy energy constants, respectively. $\theta$ is the angle between the magnetization and the applied field $H$, and $M_{S}$ is the spontaneous magnetization. The superscripts $h$ and $s$ denote the hard and soft layer, respectively. For simplicity, the easy axes of all layers are assumed to orient along the applied field direction. For a trilayer system with a sufficiently thick hard layer, the angular solutions for the hard and soft layer can be decoupled. ${ }^{4}$ Thus calculations can be performed only for a region defined

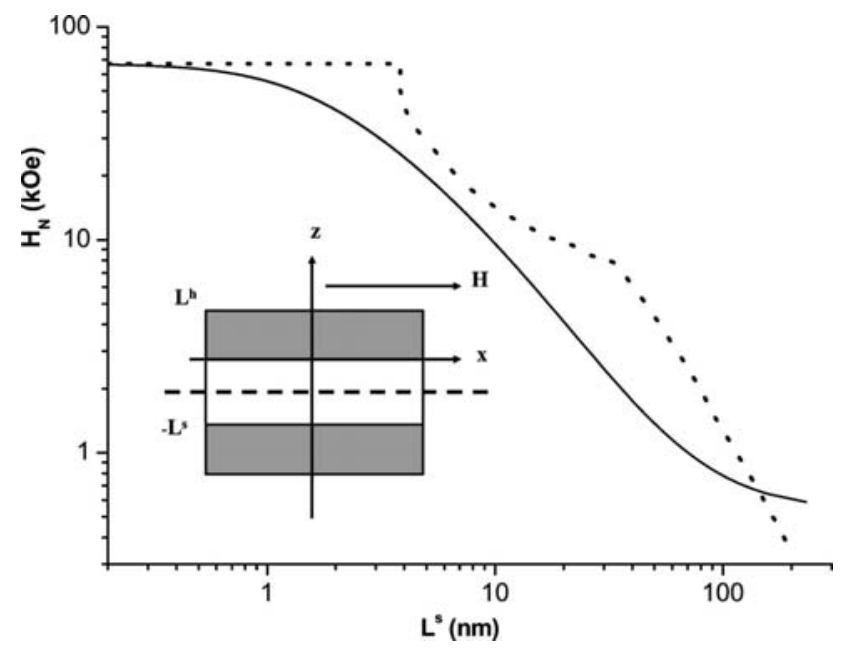

FIG. 1. Calculated nucleation fields for a $\mathrm{Nd}_{2} \mathrm{Fe}_{14} \mathrm{~B} / \mathrm{Fe} /$ $\mathrm{Nd}_{2} \mathrm{Fe}_{14} \mathrm{~B}$ trilayer system (shown in the inset) as a function of the soft layer thickness. Solid lines: present result according to Eq. (5); dotted line: numerical nucleation field from Ref. 9. 
by $-L^{s} / 2 \leqslant z \leqslant 0$ with the symmetry of the system considered.

By applying the variational method to energy (1) with suitable boundary conditions, ${ }^{4}$ we obtain the following equations for the angular distribution of the magnetization in the soft film and interface ${ }^{4}$

$$
\begin{aligned}
\frac{\pi\left(L^{s} / 2+z\right)}{\Delta^{s}}= & \int_{\theta^{0}}^{\theta^{s}} \frac{d \phi}{\sqrt{\left(\sin ^{2} \phi-\sin ^{2} \theta^{s}\right)-2 h^{s}\left(\cos \phi-\cos \theta^{s}\right)}}, \\
& -\frac{L^{s}}{2} \leqslant z<0,
\end{aligned}
$$

$$
\begin{aligned}
A^{s} K^{s} & {\left[\left(\sin ^{2} \theta^{0}-\sin ^{2} \theta^{s}\right)-2 h^{s}\left(\cos \theta^{0}-\cos \theta^{s}\right)\right] } \\
& =A^{h} K^{h}\left[\sin ^{2} \theta^{0}-2 h^{\mathrm{h}}\left(\cos \theta^{0}-1\right)\right],
\end{aligned}
$$

where $\theta^{0}$ and $\theta^{s}$ are the directions of magnetization at $z=0$ and $z=-L^{S / 2}$, respectively. The reduced applied field $h$ $=H / H_{K}$ is introduced with $H_{K}=2 K / M_{S}$ as the anisotropy field. $\Delta=\pi \sqrt{A / K}$ is the Bloch wall width. Solving Eqs. (2) and (3) will yield the nucleation and coercive fields.

The nucleation field can be derived analytically. At the nucleation state, the deviation of the magnetization from the applied field direction is small. Thus the nucleation problem can be solved by an appropriate ansatz or series expansion. ${ }^{6}$ Here, we adopt the latter method since the physics are more transparent. First, a Taylor series expansion of Eq. (3) at $\theta$ $=0$ and omitting the high-order term gives

$$
\left(\frac{\theta^{s}}{\theta^{0}}\right)^{2}=1-\frac{A^{h} K^{h}\left(1+h_{N}^{h}\right)}{A^{s} K^{s}\left(1+h_{N}^{s}\right)},
$$

where $h_{N}=-H_{N} / H_{K}$ is the reduced nucleation field. A minus symbol is adopted here since we are investigating the nucleation corresponding to the second quadrant hysteresis loop. Equation (4) demonstrates that the phase transition from a coherent state to an incoherent state for both the hard and soft layers occurs simultaneously, i.e., taking place at the nucleation field $H_{N}$. Substituting Eq. (4) into Eq. (2), after a series expansion and a subsequent integration, yields

$$
\frac{\pi L^{s}}{2 \Delta^{s}}=\frac{\tan ^{-1} \frac{\sqrt{A^{h} K^{h}\left(1+h_{N}^{h}\right)}}{\sqrt{A^{s} K^{s}\left(-1-h_{N}^{s}\right)}}}{\sqrt{-h_{N}^{s}-1}} .
$$

Equation (5) is a general formula that relates the nucleation field to $L^{s}$ and material parameters. From this relationship, some more specific formulas can be derived. For example, taking $A^{s}=A^{h}, M_{S}^{s}=M_{S}^{h}$, and $K^{s}=0$, we have $\frac{\pi L^{s}}{2 \Delta^{h}}=\frac{\cos ^{-1} \sqrt{-h_{N}^{h}}}{\sqrt{-h_{N}^{h}}}$, which is the formula of the nucleation field derived by Aharoni [Eq. (12) of Ref. 3].

Figure 1 shows the calculated nucleation fields based on Eq. (5) for an $\mathrm{Nd}_{2} \mathrm{Fe}_{14} \mathrm{~B} / \alpha-\mathrm{Fe} / \mathrm{Nd}_{2} \mathrm{Fe}_{14} \mathrm{~B}$ trilayer as a function of the soft layer thickness $L^{s}$. The material parameters are listed in Table I. As $L^{s}$ increases from 0 to infinity, $H_{N}$ decreases smoothly from $H_{K}{ }^{h}$ to $H_{K}{ }^{s}$. The numerical result by Leineweber and Kronmüller ${ }^{11}$ has been shown for com-
TABLE I. Magnetic properties for various hard and soft magnetic materials adopted from Refs. 2, 4, and 6.

\begin{tabular}{cccccc}
\hline \hline Material & $M_{S}(\mathrm{kG})$ & $\begin{array}{c}K\left(\times 10^{7}\right. \\
\left.\mathrm{erg} / \mathrm{cm}^{3}\right)\end{array}$ & $\begin{array}{c}A\left(\times 10^{7}\right. \\
\mathrm{erg} / \mathrm{cm})\end{array}$ & $\begin{array}{c}\Delta \\
(\mathrm{nm})\end{array}$ & $H_{K}(\mathrm{kOe})$ \\
\hline $\mathrm{Nd}_{2} \mathrm{Fe}_{14} \mathrm{~B}$ & 1.28 & 4.3 & 7.7 & 4.2 & 67.2 \\
$\mathrm{Sm}_{2} \mathrm{Fe}_{17} \mathrm{~N}_{3}$ & 1.23 & 12 & 10.7 & 3.0 & 195 \\
$\mathrm{SmCo}_{5}$ & 0.84 & 17.1 & 12. & 2.6 & 407 \\
$\alpha-\mathrm{Fe}$ & 1.71 & 0.046 & 25. & 73.2 & 0.54 \\
$\mathrm{Co}$ & 1.43 & 0.43 & 10.3 & 15.4 & 6.0 \\
\hline \hline
\end{tabular}

parison. Their calculation is roughly consistent with ours. However, the outstanding discrepancies around $L^{s}=4 \mathrm{~nm}$ and $33 \mathrm{~nm}$ should be noted.

For $L^{s} \leqslant \Delta^{h}(4.2 \mathrm{~nm})$, their nucleation field $H_{N}$ is independent of $L^{s}$ and identical with $H_{K}{ }^{h}$. Similar results have been obtained by Kneller ${ }^{5}$ and by Skomski and Coey ${ }^{6}$ for a spherical soft inclusion. Our analytical result, however, shows that $L^{s}=\Delta^{h}$ is not an inflection point at all and there is a rapid decrease of nucleation field with $L^{s}$ for $L^{s} \leqslant \Delta^{h}$. Similar results have been obtained by Aharoni for a more specific defect. $^{3}$ It should be noted that there are some misunderstandings in this important field due to the ambiguity of the coercivity mechanism. Recently an "effective anisotropy,"6,10 which is basically the average anisotropy of the soft and hard phases, has been used to investigate the coercivity related problems. According to this approach, the nucleation field at small $L^{s}$ should change with $L^{s}$ linearly. For very thin soft layer, Eq. (5) reduces to $H_{N}=H_{K}^{h}\left[1-\left(\frac{\pi L^{s}}{2 \Delta^{h}} \frac{M_{S}^{s}}{M_{S}^{h}}\right)^{2}\right]$. The nucleation field approaches $H_{K}^{h}$ parabolically rather than linearly. Thus the average anisotropy is not a suitable explanation for the coercivity of composite magnets. Physically this parabolic decrease of nucleation field at small $L^{s}$ implies the destructive role of the defects in reducing the coercivity and partially solves the Brown's coercivity paradox.

For $L^{s} \geqslant 8 \Delta^{h}$, the nucleation field $H_{N}$ given by Ref. 11, falling off as $\left(L^{s}\right)^{-1.75}$, is significantly smaller than in our calculation, because the authors have ignored completely the contribution from the anisotropy of the soft phase, $K^{s}$. Such an approximation, however, is only valid for very small $L^{S}$.

For intermediate $L^{s}, H_{N}$ by Ref. 11 is larger than our result, with the difference increasing with $L^{s}$. We believe that they have calculated coercivity rather than the nucleation field due to the uncertainty of the coercivity mechanism as can be seen more explicitly from the following discussion.

For arbitrary applied fields between nucleation and coercivity, the angular distribution could be obtained numerically. Such a distribution, exhibiting a three-step magnetization reversal process, has been calculated and shown in Ref. 4. Figure 2 shows the calculated $L^{s}$ dependence of the nucleation field and coercivity of an $\mathrm{Nd}_{2} \mathrm{Fe}_{14} \mathrm{~B} / \alpha$ - $\mathrm{Fe} / \mathrm{Nd}_{2} \mathrm{Fe}_{14} \mathrm{~B}$ trilayer. The numerical nucleation field in Fig. 2 is identical to the analytical one shown in Fig. 1 throughout all $L^{s}$, justifying our calculation. When $L^{s}$ is less than a certain critical thickness, $L_{c r i t 1}$, where $H_{c}=H_{N}$, the three steps of magnetic reversal converge into one single step and the coercivity mechanism is totally nucleation. As a result, the whole sys- 


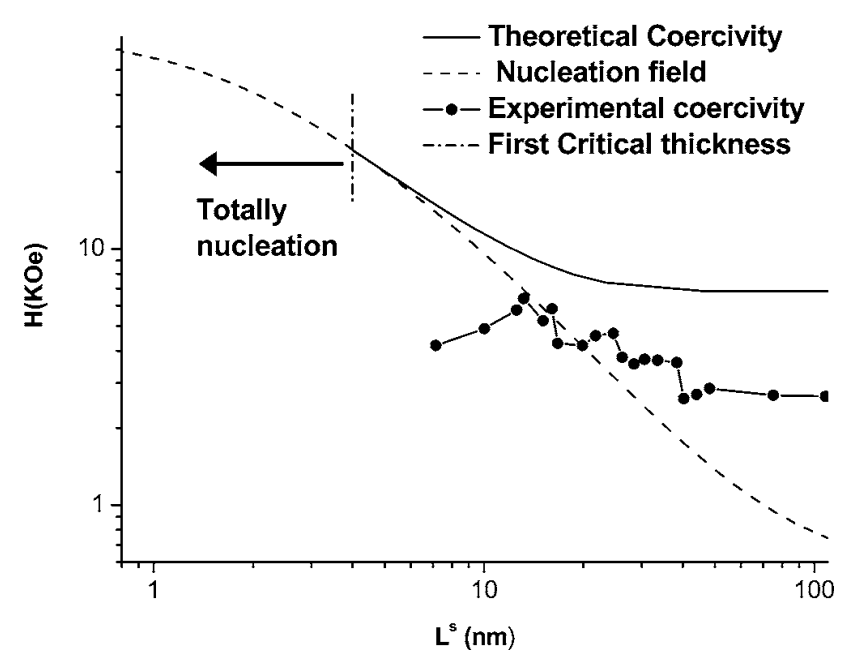

FIG. 2. Comparison of nucleation field with numerical and experimental coercivity for a $\mathrm{Nd}_{2} \mathrm{Fe}_{14} \mathrm{~B} / \mathrm{Fe} / \mathrm{Nd}_{2} \mathrm{Fe}_{14} \mathrm{~B}$ trilayer system.

tem reverses simultaneously, and there is no stable angular solution for Eqs. (2) and (3) except $\theta \equiv 0$ and $\theta \equiv \pi$. Thus the nucleation field in this region is very difficult to determine accurately, which is probably the reason for the outstanding discrepancy in the calculated nucleation field for small $L^{s}$ observed in Fig. 1. From Fig. 2, $L_{\text {crit } 1}$ of an $\alpha$-Fe interlayer sandwiched between two $\mathrm{Nd}_{2} \mathrm{Fe}_{14} \mathrm{~B}$ layers can be determined accurately as $4 \mathrm{~nm}$.

For larger $L^{s}$, there is a gap between the two fields, indicating a process of domain wall evolution near the interface, which links the nucleation and the coercivity. ${ }^{4}$ The width of the gap, $H_{c}-H_{N}$, increases with $L^{s}$, suggesting that the coercivity mechanism is changing from nucleation to pinning. For sufficiently large $L^{s}, H_{c}$ saturates at $6.82 \mathrm{kOe}$, and the coercivity mechanism changes completely to pinning. The pinning field can be derived analytically.

For large $L^{s}$, a $180^{\circ}$ domain wall forms before the irreversible domain wall motion. ${ }^{4}$ In this case the pinning field could be obtained analytically. Substituting $\theta^{s}=180^{\circ}$ into Eq. (3) yields

$$
-H=\frac{\left(A^{h} K^{h}-A^{s} K^{s}\right) \sin ^{2} \theta}{A^{s} M^{s}\left(1+\cos \theta^{0}\right)+A^{h} M^{h}\left(1-\cos \theta^{0}\right)} .
$$

By maximizing $-H$ with respect to $\cos \theta^{0}$, the pinning field is obtained as

$$
H_{p}=\frac{2\left(A^{h} K^{h}-A^{s} K^{s}\right)}{\left(\sqrt{A^{s} M^{s}}+\sqrt{A^{h} M^{h}}\right)^{2}} .
$$

Thus the pinning field is completely determined by the interface constraint. A similar result has been obtained by Kronmüller and Goll, ${ }^{12}$ but by using a different method. Taking $A^{s} \approx A^{h}, M^{s} \approx M^{h}, K^{S} \approx K^{h}$, Eq. (7) reduces to $\frac{H_{p}}{H_{K}}=\frac{\delta A / A+\delta K / K}{4}$, where $\delta A=A^{h}-A^{s}$ and $\delta K=K^{h}-K^{s}$. This is the formula for the pinning force ${ }^{2}$ exerted upon a domain wall that penetrates from a phase with low $A$ and $K$ into a phase with high $A$ and $K$. On the other hand, for a soft planar defect with $A^{s}=A^{h}$, $M^{s}=M^{h}$, and $K^{S}=0$ in a hard magnetic material, Eq. (7) re- duces to $H_{p}=\frac{H_{K}^{h}}{4}$, which has been investigated by Aharoni. ${ }^{3}$ According to Aharoni, such a defect can reduce the coercivity by no more than a factor of 4 compared to that of the perfect material and is unable to explain the experimental coercivity of one or two orders of magnitude smaller. Since $A^{s} M^{s}$ is usually larger than $A^{h} M^{h}$, according to Eq. (7), the soft defect can reduce the coercivity more efficiently. If we ignore the $K^{s}$, which is about two orders of magnitude smaller than $K^{h}$, Eq. (7) can be rewritten as

$$
H_{p}=\frac{H_{K}^{h}}{(1+\sqrt{\eta})^{2}},
$$

where $\eta=\frac{A^{s} M^{s}}{A^{h} M^{h}}$. Taking $\mathrm{Nd}_{2} \mathrm{Fe}_{14} \mathrm{~B}$ as an example, due to the existence of $\alpha$-Fe as a soft defect, $\eta=4.33$ (see Table I), the coercivity is roughly $10 \%$ of that of a perfect material. The coercivity could be less if the change of the parameters from the soft to hard phases is more gradual. ${ }^{4}$ Thus our formulas offer a possibility to further solve the coercivity paradox.

The second critical thickness, $L_{c r i t}$, is defined at the thickness where $H_{N}=H_{p}$. Beyond this thickness the dominant coercivity mechanism changes from nucleation to pinning. For an $\mathrm{Nd}_{2} \mathrm{Fe}_{14} \mathrm{~B} / \alpha$-Fe system, $L_{\text {crit } 2}=13 \mathrm{~nm}$.

Such a change of coercivity mechanism with the soft layer thickness is confirmed by comparison of experimental coercivity with the nucleation field. For $13 \mathrm{~nm}<L^{s}$ $<20 \mathrm{~nm}$, the experimental data by Parhofer ${ }^{8}$ agree very well with the theoretical $H_{N}$, indicating that the coercivity mechanism in this regime is totally nucleation. For larger $L^{s}$ there is a gap between $H_{c}$ and $H_{N}$, which increases with the $L^{s}$, demonstrating a change of coercivity mechanism from nucleation to coercivity. For $L^{s}>40 \mathrm{~nm}$, the experimental coercivity reaches a constant, showing that the domain wall pinning is the dominant mechanism. However, we note that the experimental critical thicknesses are about 20 and $40 \mathrm{~nm}$, much larger than the theoretical ones while the experimental coercivity is much smaller than the theoretical pinning field. This discrepancy is attributed mainly to the abrupt change of the material parameters at the interface assumed in Eq. (3).

On the other hand, for $L^{s}<13 \mathrm{~nm}$, the experimental coercivity decreases as $L^{s}$ decreases, which is contrary to the theoretical predictions. This discrepancy is important as the giant theoretical energy product had been thought to be achieved in this regime. ${ }^{6}$ A close inspection of the experiment shows that as $L^{s}$ decreases, $L^{h}$ also decreases. The crystalline anisotropy decreases significantly as $L^{h}$ decreases to less than $10 \mathrm{~nm} .{ }^{10}$ In addition, the interface anisotropy might affect the coercivity in this thickness regime. (The interface anisotropy, if considered, will decrease the in-plane anisotropy.) Taking account of these effects the optimum thicknesses for the soft and hard layers of a multilayer to achieve the maximum energy product are about $15 \mathrm{~nm}$ (rather than 9 and $2 \mathrm{nms}$, as given by Ref. 6) with the volume occupation of the soft phase at $50 \%$. With this percentage, the maximum attainable energy product of an $\mathrm{Nd}_{2} \mathrm{Fe}_{14} \mathrm{~B} / \mathrm{Fe}$ system is about $85 \mathrm{MGOe}\left(680 \mathrm{MJ} / \mathrm{m}^{3}\right)$ with the corresponding $M_{r}$, about $1.5 \mathrm{kG}$. This value is larger than that of the single-phase $\mathrm{Nd}_{2} \mathrm{Fe}_{14} \mathrm{~B}$, but much smaller than that given by Ref. 6 . 
TABLE II. Calculated critical thicknesses of the soft phase for various groups of hard/soft materials.

\begin{tabular}{|c|c|c|c|c|c|c|}
\hline \multirow[b]{2}{*}{ Interface } & \multirow[b]{2}{*}{$A^{s} M^{s} / A^{h} M^{h}$} & \multicolumn{2}{|c|}{ Critical fields $\left(H_{K}^{h}\right)$} & \multicolumn{2}{|c|}{$\begin{array}{c}\text { Critical } \\
\text { thicknesses (nm) }\end{array}$} & \multirow{2}{*}{$\begin{array}{c}\text { Dominant } \\
\text { coercivity } \\
\text { mechanism }\end{array}$} \\
\hline & & $h_{1}$ & $h_{2}$ & $L_{\text {crit } 1}$ & $L_{\text {crit } 2}$ & \\
\hline $\mathrm{Nd}_{2} \mathrm{Fe}_{14} \mathrm{~B} / \alpha-\mathrm{Fe}$ & 4.33 & 0.36 & 0.10 & 4.0 & 13 & Nucleation \\
\hline $\mathrm{SmCo}_{5} / \mathrm{Co}$ & 1.46 & 0.50 & 0.20 & 1.2 & 2.9 & Pinning \\
\hline $\mathrm{Sm}_{2} \mathrm{Fe}_{17} \mathrm{~N}_{3} / \mathrm{Fe}$ & 3.25 & 0.4 & 0.13 & 2.3 & 6.8 & Both \\
\hline
\end{tabular}

Similar experimental results have been obtained by other groups. ${ }^{7,9}$ For example, Shindo et al. ${ }^{9}$ found that the coercivity of the $\mathrm{Nd}_{2} \mathrm{Fe}_{14} \mathrm{~B} / \mathrm{Fe}$ multilayers reaches a peak when the thickness is $10 \mathrm{~nm}$ while it is a constant when $L^{s}$ is larger than $30 \mathrm{~nm}$, demonstrating the same change of the coercivity mechanism with $L^{s}$ as that of Ref. 8. Calculation of other magnetic systems shows similar trends in the change of the coercivity mechanism with $L^{s}$. However, the critical thickness differs from material to material. By considering the fourth order term of $\theta$ in Eq. (1), the first critical thickness can be determined analytically. ${ }^{3}$ The energy change at nucleation is given by

$$
\delta E=B\left[v \tan v-\sin ^{2} v \cos 2 v-\frac{2}{\eta} \cos ^{4} v\right] \theta^{4},
$$

where $B$ is positive and $v=\frac{\pi L^{s}}{2 \Delta^{s} \sqrt{-h^{s}}}$. If $\delta E>0$, the energy change at the nucleation is continuous and vice versa. Setting $\delta E=0$, we obtained the first critical thickness for various materials, shown in Table II. It can be seen that both $L_{\text {crit } 1}$ and $L_{\text {crit } 2}$, of the $\mathrm{SmCo}_{5} / \mathrm{Co}$ system are much smaller than those of the $\mathrm{Nd}_{2} \mathrm{Fe}_{14} \mathrm{~B} / \mathrm{Fe}$ system. This explains why the dominant coercivity mechanism in $\mathrm{SmCo}_{5}$ is pinning while that of the $\mathrm{Nd}_{2} \mathrm{Fe}_{14} \mathrm{~B}$ is nucleation. However, while the critical fields of $\mathrm{Nd}_{2} \mathrm{Fe}_{14} \mathrm{~B}$ shown in Table II are in reasonable agreement with the experimental data, the pinning field of $\mathrm{SmCo}_{5}$ is much larger because of the existence of the second hard phase in SmCo material. It can be seen from Eq. (7) that the existence of a second hard phase such as $\mathrm{Sm}_{2} \mathrm{Co}_{17}$ which is relatively softer compared with $\mathrm{SmCo}_{5}$ provides a large " $K$ " for Eq. (7) and thus decreases the pinning field significantly.

From the above discussion we can see that the relation between the coercivity and the anisotropy of the hard and soft phases depends on the coercivity mechanism. For large $L^{s}$, the coercivity mechanism is pinning. According to Eq. (7), the coercivity decreases rather than increases with $K^{s}$, proving the invalidity of the effective anisotropy explanation.

\section{ACKNOWLEDGMENT}

X.L.W. thanks the Australian Research Council for financial support through an ARC Discovery Project (DP0558753).
*Electronic address: zhaogp@uestc.edu.cn; zapple2004@yahoo.com

${ }^{\dagger}$ Electronic address: xiaolin@uow.edu.au

${ }^{1}$ W. F. Brown, Jr., Rev. Mod. Phys. 17, 15 (1945); in Micromagnetics (Interscience, Wiley, New York, 1963).

${ }^{2}$ H. Zijlstra, in Ferromagnetic Materials, edited by E. P. Wohlfarth and K. H. J. Buschow (Elsevier Science Publications, NorthHolland, Netherlands, 1996), Vol. 3, pp. 39-104.

${ }^{3}$ A. Aharoni, Phys. Rev. 119, 127 (1960).

${ }^{4}$ G. P. Zhao, M. G. Zhao, H. S. Lim, Y. P. Feng, and C. K. Ong, Appl. Phys. Lett. 87, 162513 (2005).

${ }^{5}$ E. F. Kneller, IEEE Trans. Magn. 27, 3588 (1991).

${ }^{6}$ R. Skomski and J. M. D. Coey, Phys. Rev. B 48, 15812 (1993).
${ }^{7}$ I. A. Al-Omari and D. J. Sellmyer, Phys. Rev. B 52, 3441 (1995).

${ }^{8}$ S. M. Parhofer, J. Wecker, C. Kuhrt, G. Gieres, and L. Schultz, IEEE Trans. Magn. 32, 4437 (1996).

${ }^{9}$ M. Shindo, M. Ishizone, A. Sakuma, H. Kato, and T. Miyazaki, J. Appl. Phys. 81, 4444 (1997).

${ }^{10}$ J. Arcas, A. Hernando, J. M. Barandiaran, C. Prados, M. Vazquez, P. Marin, and A. Neuweiler, Phys. Rev. B 58, 5193 (1998); G. B. Han, R. W. Gao, S. Fu, W. C. Feng, H. Q. Liu, W. Chen, W. Li, and Y. Q. Guo, Appl. Phys. A 81, 579 (2005).

${ }^{11}$ T. Leineweber and H. Kronmüller, Phys. Status Solidi B 201, 291 (1997); J. Magn. Magn. Mater. 176, 145 (1997).

${ }^{12}$ H. Kronmüller and D. Goll, Physica B 319, 122 (2002). 